\title{
Using an Individual-Centered Approach to Gain Insights From Wearable Data in the Quantified Flu Platform: Netnography Study
}

Bastian Greshake Tzovaras ${ }^{1,2}, \mathrm{PhD}$; Enric Senabre Hidalgo ${ }^{1}$, PhD; Karolina Alexiou, MSc; Lukaz Baldy, BSc; Basile Morane $^{3}$, MSc; Ilona Bussod ${ }^{4}$, BSc; Melvin Fribourg ${ }^{4}$, BSc; Katarzyna Wac ${ }^{5}, \mathrm{PhD}$; Gary Wolf ${ }^{6}, \mathrm{MA}$; Mad Ball ${ }^{2,4}$, $\mathrm{PhD}$

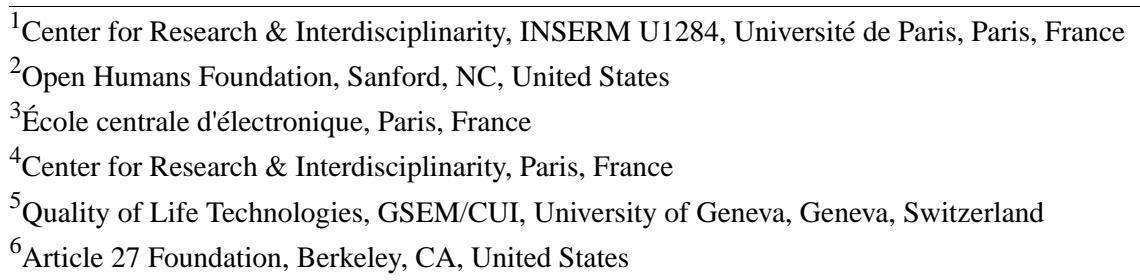

\section{Corresponding Author:}

Bastian Greshake Tzovaras, PhD

Center for Research \& Interdisciplinarity

INSERM U1284

Université de Paris

8bis Rue Charles V

Paris, 75004

France

Phone: 33766752149

Email: bgreshake@googlemail.com

\section{Abstract}

Background: Wearables have been used widely for monitoring health in general, and recent research results show that they can be used to predict infections based on physiological symptoms. To date, evidence has been generated in large, population-based settings. In contrast, the Quantified Self and Personal Science communities are composed of people who are interested in learning about themselves individually by using their own data, which are often gathered via wearable devices.

Objective: This study aims to explore how a cocreation process involving a heterogeneous community of personal science practitioners can develop a collective self-tracking system for monitoring symptoms of infection alongside wearable sensor data.

Methods: We engaged in a cocreation and design process with an existing community of personal science practitioners to jointly develop a working prototype of a web-based tool for symptom tracking. In addition to the iterative creation of the prototype (started on March 16, 2020), we performed a netnographic analysis to investigate the process of how this prototype was created in a decentralized and iterative fashion.

Results: The Quantified Flu prototype allowed users to perform daily symptom reporting and was capable of presenting symptom reports on a timeline together with resting heart rates, body temperature data, and respiratory rates measured by wearable devices. We observed a high level of engagement; over half of the users $(52 / 92,56 \%)$ who engaged in symptom tracking became regular users and reported over 3 months of data each. Furthermore, our netnographic analysis highlighted how the current Quantified Flu prototype was a result of an iterative and continuous cocreation process in which new prototype releases sparked further discussions of features and vice versa.

Conclusions: As shown by the high level of user engagement and iterative development process, an open cocreation process can be successfully used to develop a tool that is tailored to individual needs, thereby decreasing dropout rates.

(J Med Internet Res 2021;23(9):e28116) doi: 10.2196/28116

\section{KEYWORDS}

symptom tracking; COVID-19; wearable devices; self-tracking; citizen science; netnographic analysis; cocreation 


\section{Introduction}

\section{Background}

Patient- or participant-led research has been suggested to improve self-management capabilities [1] and provide ways to generate otherwise undone science [2,3]. A particular subtype of participant-led research is personal science, which involves the use of empirical methods by individuals to pursue personal health questions [4]. Personal science is a distinct category of citizen science that has emerged from the Quantified Self community and its efforts to advance participant-led research $[5,6]$. In personal science, practitioners almost always take the lead in all stages of the research process by definition [4]. Owing to this high level of individual engagement and tailoring to individuals' interests, personal science has the potential to deliver novel insights relevant to its practitioners [7], which can lead to an improved sense of agency and quality of life [8]. Furthermore, the insights and self-expertise generated by these types of participant-led processes have potential relevance for professional and scientific research, both topically as a source of ideas and methodologically as a source of tools, analytical approaches, and workflows [9].

Wearable devices - from wristbands to smartwatches and other personalized, miniaturized on- and around-body devices-are frequently used by self-trackers. These devices are becoming increasingly common and are used for a wide spectrum of well-being, fitness, and health-related purposes [10]. This is further facilitated by the fact that the number of sensors used in these devices is growing rapidly. In addition to accelerometers and gyroscopes to track physical activity, sensors to measure physiological signals such as heart rate, body temperature, respiratory rate, and blood oxygen saturation, which may correspond to the health or sickness state of the human body [11], are also frequently found in wearables [12,13]. Consequently, even outside the realms of personal science, wearables have long been seen as promising tools for facilitating health-related monitoring and enabling personalized medicine $[14,15]$ and have been proposed or used to monitor conditions as diverse as cardiovascular disease [16,17], Alzheimer [18], and graft-versus-host disease [19].

In response to the COVID-19 pandemic, interest in using wearable technology for infection prediction and surveillance has increased [20-22]. Anecdotal reports from self-trackers suggest that wearables may provide evidence of COVID-19 infection [23]. During the first year of the COVID-19 pandemic, a small number of studies appeared, highlighting that wearable devices, often along with self-reported symptoms, might indeed be used for the early detection of COVID-19 infections and to assess physiological symptoms [24-27]. The majority of these studies take a crowdsourcing-based approach-in which participants are invited to contribute by providing their own wearable data along with regular symptom reports and COVID-19 test results - as the main way of engaging individuals. The goal of the data collection process in these studies is to create big data sets to interrogate.

In contrast, there have been limited efforts to engage personal scientists in cocreating such symptom tracking efforts. Personal science practices are largely done in isolation, and the Quantified Self movement has consequently accumulated limited knowledge so far [8]. To fill this gap, we present a case study of Quantified Flu (QF), a project cocreated by a community of personal science practitioners in response to the COVID-19 pandemic.

\section{Objective}

The goals of this work are twofold. First, we documented the contrasting cocreation approach of QF with its focus on personal science rather than large-scale research. To this end, we used netnographic methods to document how the cocreation process developed and generated a citizen science platform prototype over a relatively short period. Second, we explored the consequences of the projects' contrasting cocreation approach and focused on personal science, particularly with respect to the ultimate design of the QF tool and its use.

\section{Methods}

\section{Overview}

The cocreation process of this study is based on an action research approach [28], simultaneously for developing a useful community resource while also generating shared knowledge about the process. In our case, action research was implemented through practical work to support the participatory design of a digital platform [29,30] under open-source principles [31], followed by netnographic data collection and analysis to understand its development and usefulness as a cocreation process [32]. For this, all authors except ESH were involved as participants in the cocreation process, in collaboration with the rest of the participants, during the iterative prototyping of the QF platform.

\section{Community Cocreation Process}

QF began with a discussion on the monthly Open Humans $(\mathrm{OH})$ community call at the beginning of the COVID-19 pandemic on March 10, 2020. $\mathrm{OH}$ is a platform for empowering individuals around their personal data, to explore and share research processes for the purposes of education, health, and science in general [33]. The community calls involved 83 individuals so far (until September 3, 2020), and the monthly calls are frequented by a mix of citizen science and personal science practitioners; usually, around 10 individuals take part in each call. Following an initial brainstorming, the discussions and planning stages were continued through the following community calls and a dedicated communication channel of the $\mathrm{OH}$ community Slack [34]. Furthermore, over the evolution of the project, other communities such as Quantified Self [35] and OpenCovid19 Initiative [36] were engaged and involved in different aspects of the development of the project.

In parallel to 10 additional community calls between March 10 and September 3, 2020, the main coordination tool for the QF project was a specific Slack channel, with a total of 146 subscribers and $34.2 \%(50 / 146)$ active users over time with different levels of involvement and activity. During this timeframe, this openly accessible channel gathered a total of 844 messages from these users, with a total count of 26,691 words (and 3917 unique words). 
Although the planning, coordination, and social aspects of the cocreation process mainly took place on the mentioned project's Slack channel, technical collaboration and software development occurred through GitHub and the git repository of the QF. Due to the iterative nature of open-source collaboration, no up-front requirement analysis was performed. Instead, prototypes were developed over time according to community discussions by iteratively adding and testing implementations. On GitHub, 7 contributors created a total of 316 commits since March 12, 2020, leading to the technical prototype outlined later. The source code for the project is available under an open license on GitHub [37].

\section{Netnographic Content Analysis}

To investigate and analyze the cocreation stages that led to the QF prototype, we performed a netnographic analysis of its iterative communication process, similar to previous studies on cocreation in health-related community settings [38].
Netnography is an interpretive research method derived from ethnography, usually applied to social interaction processes in digital channels and platforms, and focused on digital traces of public conversations as analyzable data. As a qualitative technique broadly applied to the study of web-based communities [32], Netnography allows capturing and reflecting interactions as an observational, inductive, and unobtrusive approach while combining it with participatory methods [39]. In particular, we examine how individuals engaged in the QF Slack channel for the collaborative development of the QF platform as a case study setting [40].

For this part of data collection, one of the researchers (ESH) developed a codebook combining key concepts of cocreation and collaboration in communities of practice (Textbox 1). The codebook was cross-checked for validity by 2 other authors (BGT and MB). Following this, it was applied to the QF Slack channel posthoc without this specific researcher (ESH) having participated in the previous community discussions.

Textbox 1. Codebook for Quantified Flu Slack communication message content analysis.

Communities of practice-related messages

- Socialization

- Support or coordination: parallel messages regarding overall coordination and personal and empathic support interventions

- Possible collaborations: ideas regarding potential collaborators and connections to other organizations or experts who can support or contribute to the project

- Outreach: messages related to the visibility of the project, possible dissemination, or alliances for spreading the process

- Off topic: nonrelated messages to any of the previous (eg, about personal issues or intention-to-buy wearables)

\section{Cocreation-related messages}

- Ideas

- Inspiring or similar initiatives: mentions to other COVID-19-related projects being developed or known externally

- COVID-19 related: links to news or updates regarding the COVID-19 pandemic and its evolution

- Mention to tool or wearable: references to a specific wearable for its potential connection to the Quantified Flu project

- Scientific knowledge or papers: mentions or links to studies or publications and elaborated scientific knowledge

- $\quad$ The Quantified Flu concept

- Goal setting or discussion: concept-related interventions about the objectives of the project

- Protocol or tool design: mentions to how the protocol and tool should work or specific aspects of its possible design

- Feature suggestion: interventions suggesting specific characteristics or new possible features of the tool

- Pattern or data observation: statements regarding the observation of data in relation to the goals or possible functioning of the project

- $\quad$ The Quantified Flu prototype

- Incremental development or updates: messages informing about new implementations and code development of features applied to the prototype

- Technical issues: specific technical issues to solve or observations about needed improvements for correct use

- Help testing: interventions asking or offering support in testing the tool by community members

- Help developing: interventions asking or offering technical support for the development of the tool

This part of data analysis was used to determine the typology of messages regarding the cocreation of the QF platform, from idea to concept to prototype [29], and other types of messages relevant from a communicational and empathy-needed dialogic process in communities of practice [41]. Each Slack message was assigned up to three top tags based on the aforementioned 
codebook categories, depending on its text density and characteristics. The researcher (ESH) assessments of types and categories of messages were subsequently reviewed and discussed by another coauthor (BGT), who was actively involved in the analyzed cocreation process.

\section{Results}

We present the current prototype of the QF platform [42], as a result of the described technical development, before analyzing the cocreation process that led to it.

\section{Community-Based Development}

The first overview derived from our netnographic analysis of the four main categories of messages interchanged during the cocreation of the QF prototypes on its dedicated Slack channel (March 10 to September 3, 2020) shows a relative balance in the topics of the web-based messages among the 1171 message fragments that were annotated (Textbox 2).

Overall, during the development of QF, the Prototyping and Socialization messages were slightly more common than the Concept and Ideas ones (Figure 1). On the level of the tags or subcategories, the most frequent ones are Support or coordination (227), Protocol or tool design (109), Technical issues (107), and Help developing (106).

Focusing on these more specific tags, as defined in the codebook (Textbox 1), within each category over time (Figure 1), we observe that all the four main categories, as well as the individual tags, are present over the whole time frame of cocreation from early April to September 2020. In particular, messages regarding Support or coordination are present throughout the entire time range. Other recurrent message types during the analyzed time span fall within the categories Ideas, Concept, and Prototyping, highlighting the iterative design, implementation, and testing participatory processes that took place to develop and improve the QF prototype over time.

Importantly, the Protocol or tool design, Mention to tool or wearable, and Feature suggestions categories, which are indicative of the cocreation process, appear early on but remain active in bursts throughout the full observed time span, often following new releases of the QF prototype. In addition, the Help developing and Help testing categories remain active over the whole duration of the prototype development, with the former showing a more constant activity (mean 1.1 tags per day, SD 1.9) whereas the latter appears in bursts (mean 0.76 tags per day, SD 2.1) around new feature releases. 
Textbox 2. Examples of messages that were tagged according to the codebook used for the netnographic analysis.

\section{Socialization}

- Support or coordination

- "Very good community call focused on quantified flu this morning. Glad I participated"

- "Great updates, thank you and I am definitely staying tuned...how can I help other than visualisations. Have a good rest of the day!"

- Possible collaborations

- " 'Person outside the community] is usually sitting in the office right next to mine (working from home these days) and we'll do a call tomorrow to chat about synergies!"

- IIt's possible we could address these with support from the company, which in turn depends on our convincing them to prioritize this support. We have some close contacts there that could lead to success"

- Outreach

- "We're (very briefly) featured in the latest UCSD newsletter"

- "Oh, and we already got some media coverage in the german 'digital living' magazine t3n"

- $\quad$ Off topic

- "Not sure if it's appropriate to ask but does anyone have a way to get discount on the Oura ring?"

- $\quad$ "BTW, semi-related to this project: Just coinciding with the general lockdown in Paris I stopped smoking and could nicely see my resting heart rate drop, my heart rate variability grow, etc. within the first few days"

Ideas

- Inspiring or similar initiatives

- "Another flu-tracking app, from Duke:...[URL]"

- "Looks like Michael Snyder's famous self-tracking lab at Stanford is doing something similar"

- COVID-19 related

- "Placing this link here because it was an interesting symptom diary someone shared on Twitter they made"

- "Btw. during the community call yesterday, [community member] shared this symptom report of a contributor who thinks he has covid19"

- Mention to tool or wearable

- "How far is the Fitbit Intraday integration? As of right now the Fitbit Graph seems quite a bit less detailed than the Oura one"

- "The Garmin devices are a bit tricky, as their API is locked off unless you apply for access with them"

- Scientific knowledge or papers

- “August 31 Webinar from hlth.com: Wearable Technology's Potential to Help Detect Illness"

- "Stanford's 2017 paper was all about longitudinal health data and health outcomes"

\section{The Quantified Flu concept}

- Goal setting or discussion

- "Personally, I see the purpose of this project not so much as epidemiological, but about expanding the personal value of our data. Doing that as a group helps us learn from context, as well as individually"

- "This project might also be a starting point for prospective tracking for people that get sick, going forward. Still thinking about if/how that would work."

- Protocol or tool design

- "So i think it may make sense to give numerical values for each symptom, from 1-5 or 1-10 in terms of intensity, and also timestamp them to allow for multiple logging within a day"

- "but my vicks smart temp thermometer arrived...the associated app allows me to record...medication, symptoms (cough, sore throat, chills, body ache, ear ache, nausea, stomach ache, fatigue, short breath, headache, diarrhea, runny nose), a free text "notes"”

- Feature suggestion 
- "She wears an Apple Watch and has resting heart rate data. Should I invite her join quantified flu even though she does not have an Oura or Fitbit? Is adding support for Apple Watch too much work at this stage? Manual entry wouldn't be very challenging probably"

- "I'm realizing the public list could at least give event IDs so you have some sort of identifier for each one"

- Pattern or data observation

- "Already contributed two sick events of mine from 2019, that are very obvious in the data but also quite different"

- 'So I don't think it's necessarily measurement noise. For my own data my gut feeling is that all variations $\leq \pm 0.3^{\circ} \mathrm{C}$ are probably just daily fluctuations for a myriad reasons"

\section{The Quantified Flu prototype}

- Incremental development or updates

- "Some publicly available data now - you can explore on the site, and there's JSON endpoints to get raw data"

- "Hey <!channel>, we have another nice visualization update thanks to [community member]! The retrospective events now have the same display that can be found for the ongoing symptom reports, check out [QF link] for an example!"

- $\quad$ Technical issues

- "Oh, not sure if that's true though! I think if the oura dies while doing the recording it doesn't deliver any data (happened to me 3-4 times with my broken oura where it would not record anything for the night)"

- "Also, I found a strange inconsistency in the data. For one of the users, the JSON file states that they are sick on July 11th, but the interactive display on the website does not (the JSON says that the person had a sore throat, but the web display does not). I attach the examples"

- $\quad$ Help testing

- "My daily symptom checkins have stopped, is this happening for anybody else? I thought it might be an email issue on my side"

- "Does anyone of you have an android watch/wearable that would track heart rate to test whether it works?"

- Help developing

- "Hi everyone! I am a programmer and would be happy to help. I have lots of experience with python"

- "I thought a cool starting visualization could be a heatmap similar to the github activity view, but with time only on the x-axis and the different symptoms on the y-axis and colored by symptom severity. If you have other cool ideas for appealing and insightful visualizations feel free to let us know!" 
Figure 1. Distribution of message types over time; the frequency of tags are given as 7-day rolling averages. Events 1-7 around Quantified Flu development are given as vertical lines. Bar plots show the total number of tags per category.

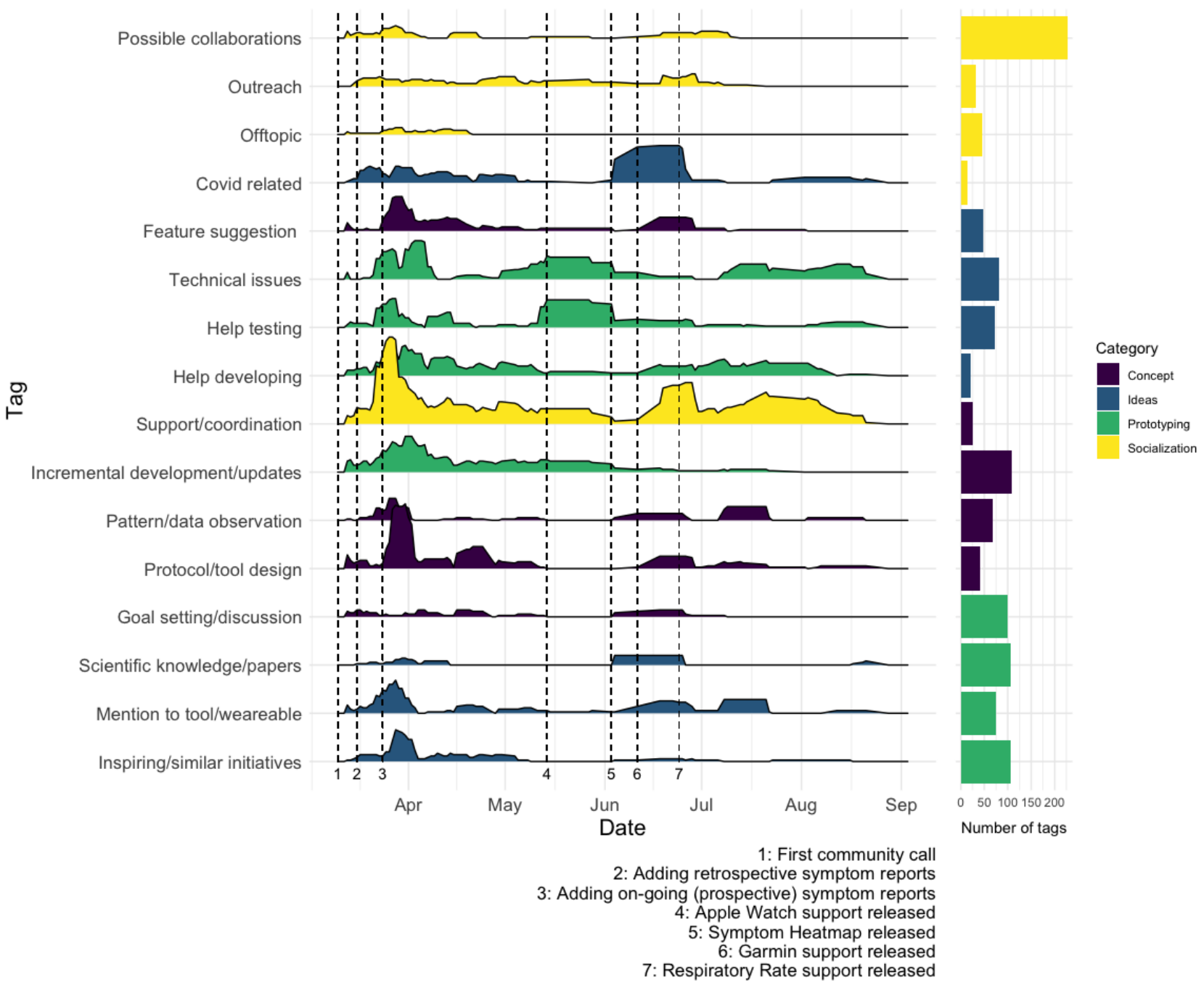

\section{QF: Technical Platform Implementation}

\section{Overview}

As a result of this community-based development process, $\mathrm{QF}$ evolved into a responsive web application that can connect to a wide variety of devices, implemented in Python or Django programming language. Users must be registered on $\mathrm{OH}$, having the option of linking a range of available wearable devices from which physiological data (heart rate, body temperature, and respiratory rate) can be imported into the $\mathrm{OH}$ platform; visualize past sickness or infection events (retrospectively) on it (present since the first prototype, launched on March 16, 2020); and engage in daily (prospective) symptom tracking (added in the second prototype, released on March 24, 2020; Figure 2). 
Figure 2. Data flow and user flow in Quantified Flu. RHR: resting heart rate.

\section{Open Humans}

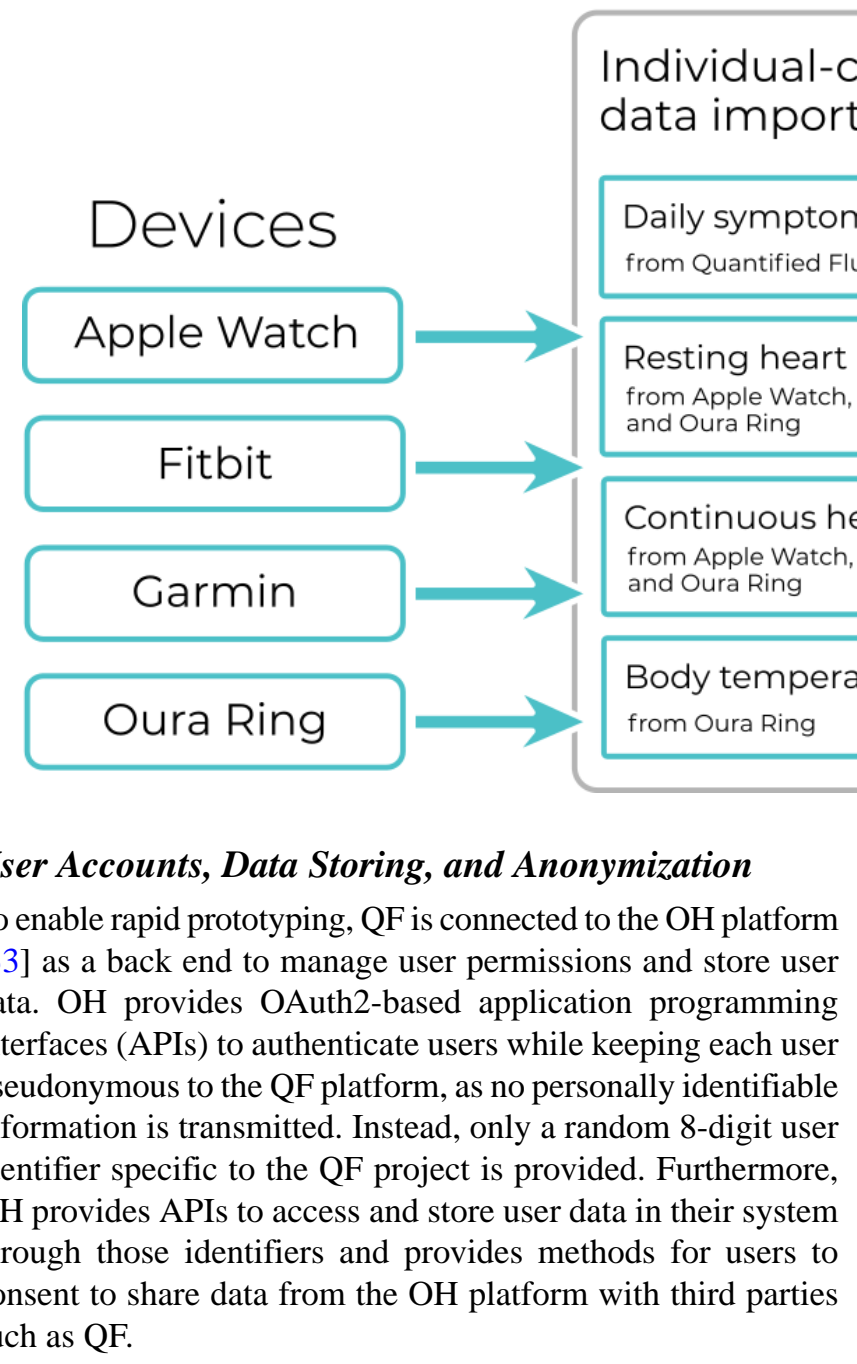

\section{User Accounts, Data Storing, and Anonymization}

To enable rapid prototyping, $\mathrm{QF}$ is connected to the $\mathrm{OH}$ platform [33] as a back end to manage user permissions and store user data. OH provides OAuth2-based application programming interfaces (APIs) to authenticate users while keeping each user pseudonymous to the QF platform, as no personally identifiable information is transmitted. Instead, only a random 8-digit user identifier specific to the QF project is provided. Furthermore, $\mathrm{OH}$ provides APIs to access and store user data in their system through those identifiers and provides methods for users to consent to share data from the $\mathrm{OH}$ platform with third parties such as QF.

\section{Wearables}

\section{Quantified Flu}

\section{Symptom Tracking}

Daily email prompt. Report no symptoms, or symptoms and/or notes.

\section{Retrospective} visualization

Plot heart rate, $\mathrm{RHR}$, and temperature for past events.

\section{Ongoing} visualization

Plot heart rate, $\mathrm{RHR}$, and temperature with symptom reports.

To further bootstrap the creation of the prototype, QF made use of the existing wearable integrations that $\mathrm{OH}$ already offered (Fitbit daily summaries, Fitbit intraday data resolution, and Oura Ring). To facilitate usability, QF also integrated these data import methods directly into the prototype, using $\mathrm{OH}$ as the data store for the wearable data.

Furthermore, following community suggestions and ideation discussions (Textbox 2; Mention of tool or wearable), QF also added Google Fit (May 6, 2020), Garmin (June 11, 2020), and Apple Health (May 14, 2020) as additional supported wearable devices. Depending on the wearables, users can import and use their heart rate throughout the day, daily resting heart rate, body temperature, and respiratory rate in $\mathrm{QF}$ (Table 1).

Table 1. Wearables supported by Quantified Flu.

\begin{tabular}{|c|c|c|c|c|c|}
\hline Wearable & Development & Resting heart rate & Heart rate throughout day & Body temperature & Respiratory rate \\
\hline Fitbit & Existing & $\sigma^{\mathrm{a}}$ & & & \\
\hline Fitbit Intraday & Existing & $\checkmark$ & $\checkmark$ & & \\
\hline Oura Ring & Existing & $\checkmark$ & & $\checkmark$ & $\checkmark$ \\
\hline Google Fit & Extended (added heart rate data) & $\checkmark$ & & & \\
\hline Apple Health & Added & $\checkmark$ & $\checkmark$ & & \\
\hline Garmin & Added & $\checkmark$ & $\checkmark$ & & \\
\hline
\end{tabular}

${ }^{\mathrm{a}}$ The feature is measured by the wearable.

Unlike other wearables integrated into QF, Apple Watch does not provide a web-based API to access and export data. Thus, following another community suggestion (Textbox 2; Feature suggestion), a mobile iOS app was created to provide a link to QF. This specific app enables users to export their heart rate data collected by Apple Watch. The source code for this mobile app is also available under an open license [43].

\section{Symptom Tracking}

Users can report symptoms using a QF website. On the basis of previous works [24-26] and early community discussion and feedback (Textbox 2; Protocol or tool design), QF implemented a list of 12 symptoms that were classified as respiratory, gastrointestinal, and systemic symptoms (Textbox 3), allowing users to score those on a 5-point scale (1=light; 5=worst). In 
addition, users can report fever measurements and use free-text fields for the suspected origin of their symptoms, further symptoms, or notes to put their symptoms into context (Textbox 2; Protocol or tool design).

Textbox 3. Symptoms of sickness that users can monitor in Quantified Flu.

\author{
Respiratory \\ - Cough \\ - Cough with mucus or phlegm \\ - $\quad$ Reduced sense of smell or anosmia \\ - Runny or stuffy nose \\ - $\quad$ Sore throat \\ - Shortness of breath \\ Gastrointestinal \\ - Diarrhea \\ - Nausea or vomiting \\ Systemic \\ - Chills and sweats \\ - Fatigue and malaise \\ - Headache \\ - $\quad$ Muscle pains and body aches
}

Users can opt in to receive daily symptom report reminders that are sent through the anonymous $\mathrm{OH}$ email system at a user-selected time, as another tool feature that was discussed and regularly tested by participants (Textbox 2; Help testing). Each email contains the following two links: (1) the reporting no symptoms link, a single-click link that requires no further interaction of the user, and (2) the reporting symptoms link, which takes users to the symptom report form.

\section{Data Visualization}

\section{Overview}

To provide users with easy ways to facilitate understanding of their own physiological data and potentially explore it in relation to their own symptom reports, QF used D3.js to create interactive visualizations. These visualizations present the evolution of the various physiological data points and put them into the context of their symptom reports where available.

The QF platform provides personal science practitioners 2 main ways to explore their physiological wearable data in relation to infections - the retrospective analysis of prior events and an ongoing (prospective) analysis of symptom reporting.

\section{Retrospective Analysis}

Users can select a given, historic date on which they fell sick or had specific symptoms, and QF will, if available, extract wearable data for the 3 weeks before that date and 2 weeks after the incident. This allows users to visualize sickness incidents that occurred before the launch of QF. Depending on the wearable (Table 1), users are given the option to display different physiological variables over that 5-week time period and explore how they change over time. To facilitate the interpretation of changes and outliers in the graphs, both the first and second SDs are presented as well (Figure 3). Although users can add comments to retrospective events, detailed symptom reports are absent in this mode, as most users do not have detailed records of the historic sickness events. The retrospective analyses were part of the first prototype of the QF, launched on March 16, 2020. 
Figure 3. Screenshots of the Quantified Flu prototype showing typical visualizations generated by users. (A) An example data visualization of an individual, retrospective sickness incident that happened on December 31, 2018. The data plotted are the resting heart rate recordings as measured by the Fitbit and Oura Ring. (B) An example of an ongoing symptom report visualization. The top half shows a heat map of the symptoms that were present along with their strength, and green boxes display user-provided free-text comments. The bottom half shows physiological data from wearables. bpm: beats per minute.

\section{A Heart rate evolution}
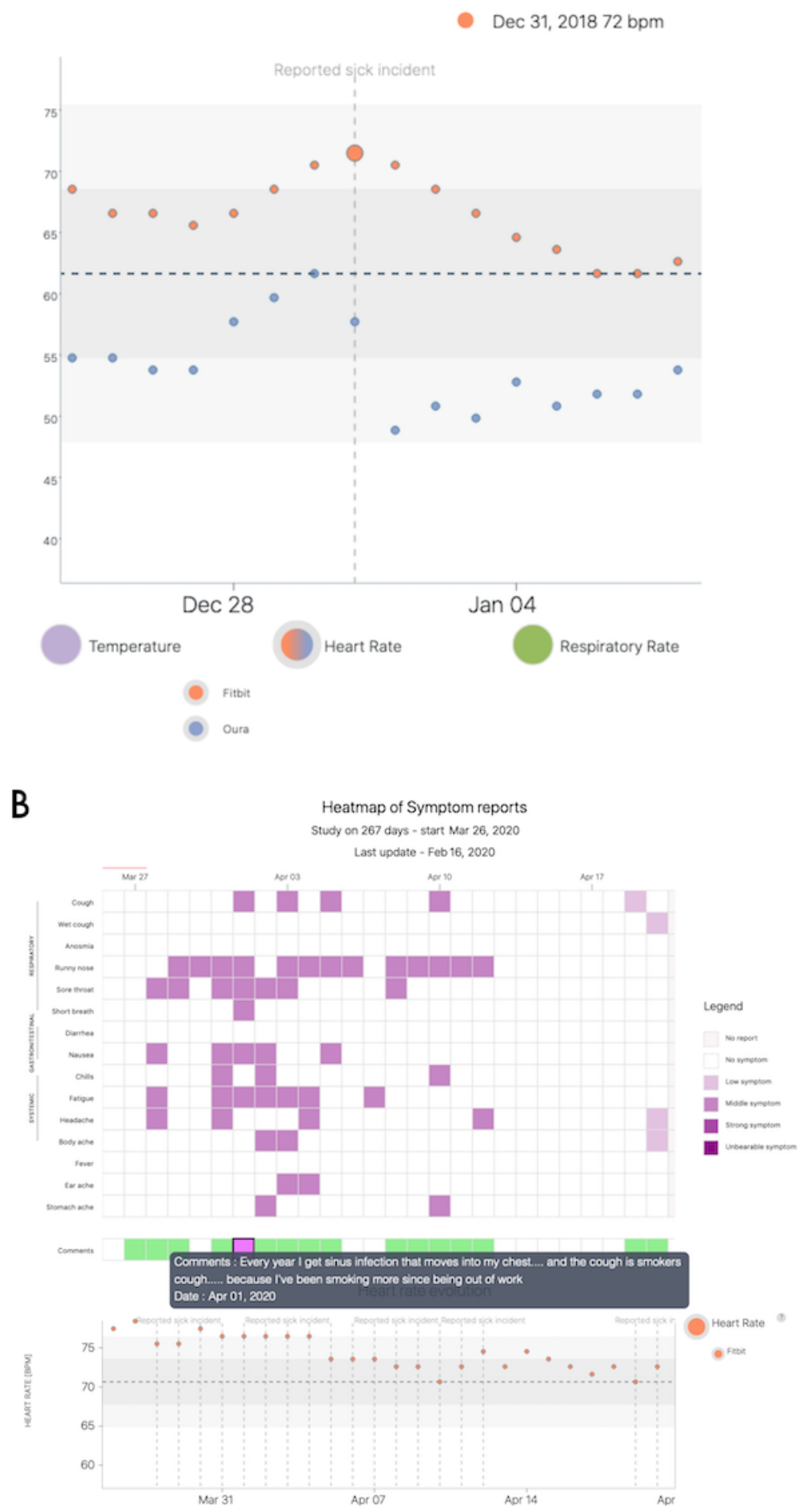

\section{Ongoing Symptom Reporting}

Users can also report currently experienced symptoms through $\mathrm{QF}$ at any moment in time by selecting symptoms and their experienced strengths from a list (Textbox 3). This self-report is likely triggered by email, as explained earlier. Following symptom reports, users are automatically taken for their data visualization (Figure 3). On a wearable device data level, this visualization provides the same details as that of the retrospective analyses (Retrospective Analysis section). The ongoing symptom reports (Figure 3) were launched as a new feature in the second iteration of the prototype on March 24, 
2020, also following discussion and contributions from the community (Textbox 2; Incremental development or updates).

In addition, this latter view aligns a heat map of each daily symptom report to the wearable data timeline, allowing the identification of patterns within the reported symptoms themselves and for visual cross-comparisons between the physiological data and the symptom reports. Furthermore, users can also access their comments for each symptom report from this visualization, allowing them to understand the contexts in which they made those reports.

\section{Community Use}

A total of 190 personal science practitioners engaged with QF between its launch on March 16, 2020, and December 22, 2020. The initial prototype of QF (in place until March 24, 2020) only offered the possibility of analyzing retrospective sickness events. This feature was rarely used: only 24 users tried the feature, creating a total of 47 retrospective analyses. In total, 34 individual wearables were linked by these 24 users. The prospective ongoing symptom report feature was launched on March 24, 2020. In total, 92 users made use of this feature at least once, covering a range from a single symptom report being done up to over 300 reports for some members. Overall, 11,658 symptom reports were filed and 112 wearables were linked to it, between the launch of the feature and December 22, 2020.

The distribution of user engagement for the entire period (Figure 4 ), as measured by the number of reports, shows an approximately linear relationship between the number of reports done and the user's rank of activity. The reports with symptoms are also not equally distributed across all 92 users, with a sizable fraction of users having no or only a few reports that include symptoms, whereas for some users, symptom reports comprise half or nearly all of the reports. Overall, the vast majority $(10,594 / 11,658,90.87 \%$ reports) were reports that included no symptoms. Of the 1064 reports with symptoms, 176 (16.54\%) included explanatory notes or comments, in addition to the standardized symptom reports.

Figure 4. The use of Quantified Flu as measured by ongoing symptom reports filed by users. (A) Users were ranked by the number of symptom reports they have filed; data were broken down into whether symptoms were reported (blue) or not reported (red). (B) The number of symptom reports filed per day. Values were averaged into a weekly rolling average. The blue line represents the local regression-smoothed data along with SE (gray background).

A
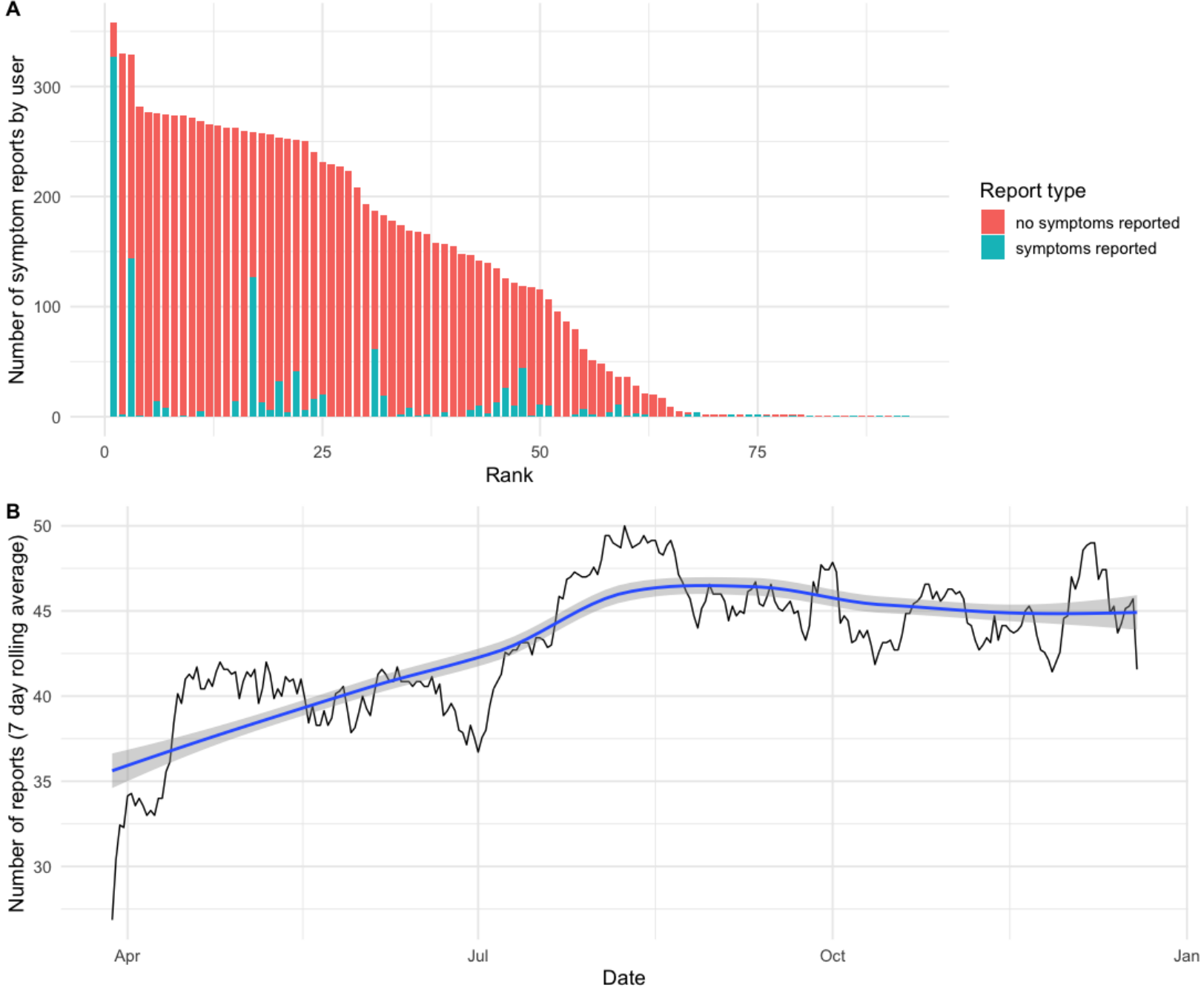

Looking at the number of symptom reports filed per day, we can observe a rapid rise in daily reports at the beginning of April ongoing symptom reports. The second rise in daily reports 2020, reflecting the launch of the first prototype with the started in July 2020, leading to the numbers starting to stabilize 
at around 45 symptom reports filed per day (by an average 45 users per day, SD 5; Figure 4).

\section{Discussion}

\section{Principal Findings}

In this paper, we present QF, a cocreated web-based project that enables personal science practitioners to engage with their own wearable data and visualize it in the context of when they are experiencing symptoms of potential infection. The spark that led to this community deciding to cocreate a symptom tracking tool was the beginning of the global COVID-19 pandemic, along with population-wide studies that made individuals wonder how useful their own wearable data might be for them in such a pandemic context. With its focus on individual learning, QF stands in contrast to various population-level studies performed to evaluate the usefulness of wearable technology for the prediction of illness [20,24-27]. At this individual scale, symptom tracking and health data more generally can offer support for individual sense-making on health experiences and conditions [44], which can be idiosyncratic and complex [45]. QF was also distinguished by a cocreation approach that targeted the individual learning and research interests of a web-based community and involved the iterative development of a digital tool in response to feedback, resulting in a format that attracted increased and sustained participation from early users.

One of the main aims of our work is to investigate the consequences of a cocreation approach that focuses on personal science: We observe that the initial QF prototype, which focused solely on retrospective symptom tracking, was rarely used. Only 24 users were engaged in this prototype. However, importantly, this initial version facilitated additional discussions about designing both the data collection protocol and extending the prototype (Figure 1), leading to the creation of the ongoing symptom reports as a feature launched in the next QF iteration. This feature received much more attention from the participants, with a total of 92 people using QF for their own regular symptom tracking, delivering some first insight into the importance and potential benefits of early engaging potential users in a health research design cocreation approach.

Furthermore, we observe that the level of engagement across these 92 QF users seems to drop linearly when ordered from most to least engaged users (Figure 4). This distribution is atypical for user engagement in web-based communities, where one typically observes power-law distributions for engagement [46]. Related to this, digital or mobile health apps in particular typically struggle with achieving continued use, as a large fraction of users drop out after a few interactions [47,48]. In previous studies, only $2 \%$ of initial users showed sustained use in the most extreme cases, with observational studies having an average dropout rate of $49 \%$ [49]. In contrast, around half of the QF users who engaged with ongoing symptom tracking did so on a regular basis, leading to 45 (SD 5) symptom reports per day on average (Figure 4), and over 50 users reported more than 3 months of symptom reports, highlighting continued longitudinal use. We argue that these uncharacteristically high numbers of user engagement, which is sustained over time, is a result of the community cocreation process that led to the final prototype of QF. Previous studies have found that users are more likely to continue using mobile health apps if there is a good fit between users and applications [50], which means that a cocreation process among future users could be a key way of achieving this fit.

For some users, this continued engagement might also be an indication that they experience regular or recurring symptoms, making them particularly interested in learning empirically about them through this specific kind of self-tracking. This is supported by the number of reports that include symptoms, where a subset of users reported having symptoms frequently, with some users reporting symptoms in $40 \%$, or extreme cases even $90 \%$, of the time (Figure 4). Further evidence for this comes from the notes or annotations that users can submit to the QF website along with their symptoms when filing their daily reports. Looking at the publicly shared notes in these reports, we find examples like "the cough is smokers cough...because I've been smoking more since being out of work" and "I was deep cleaning the house....all the dust got my allergies going again" highlighting possible reasons for recurring symptoms. Furthermore, these annotations help to provide context to individuals and others that aim to reuse publicly shared data. Although a severe case of coughing or nasal congestion might hint at acute infection, they might also be unrelated, as the annotations highlight. These contextual descriptions can be difficult to formalize, potentially explaining why symptom-based diagnoses are difficult to achieve in many cases [51,52].

Our second main goal, in parallel to the development of the QF prototype itself, is to explore how a community-driven initiative can contribute to collectively creating the tools needed to build self-knowledge by conducting a netnographic analysis of the main QF communication channel. Reflecting on the use of this qualitative and interpretative methodology for the study of web-based communities [53], we find that it adapts well to user-led prototypes, with some particular strengths and limitations. In the case of QF, we found that the netnographic approach was well suited to allow a posthoc study of the participatory design process after the prototype creation. This approach could be valuable in obtaining a better understanding of cocreation dynamics in similar health-related projects and studies [54], as it can be applied to existing text corpora of community interactions on digital text tools such as Slack, mailing lists, or forums. Its reliance on text communication is also one of the main limitations, as synchronous meetings_-remote and physical—are less accessible as archival data, requiring recordings and transcriptions. Given this, it might be advisable to organize cocreation processes with Netnography techniques in mind to ensure adequately sized text corpora.

Applying such a netnographic approach to QF, we found a marked overlap of the various phases of ideation, conceptualization, and prototyping over time. Although a greater number of interactions can be found in the initial phases, there is a sustained regularity later on, particularly in areas such as feature suggestions or the design of the tool and protocol. In this sense, messages and interactions related to helping with development throughout the whole process reflect a typology of continuous and iterative cocreation, which is typical of 
collaboration processes in the development of open-source tools [55].

This iterative cocreation process is also highlighted in the burst-like appearance of feature suggestions and protocol or tool design discussions, which frequently appear following the release of new features, suggesting that new releases spark further protocol refinements and feature ideas, which in turn lead to the QF prototype refinement. Importantly, this means that the protocol itself, along with the concrete implementation, remains in a stage of flux over a longer period of time, compared with more traditional research design approaches. As a result, this type of collaborative approach is at odds with standard ethical oversight procedures for human subject research that require a precise predefinition of the protocol and the role of the individuals, whereas the main feature of cocreation is that it is emergent and adaptive, making detailed prespecifications impossible [56]. To fully take the advantage of the benefits of cocreation in the participant-led research, it might be necessary to develop different models of ethical oversight that recognize the autonomy of participants [57,58], to not discourage or stifle valuable forms of participant-led research [2].

Finally, it is also important to highlight how the other types of messages associated with communication in a community of practice context, which favor both web-based empathy and effective coordination, were produced in a prominent, constant, and sustained manner from the beginning of the cocreation process (Support or coordination; Figure 1). This mode of cocreation can be understood as an example of uninvited citizen science that relies on a shared set of values, self-stabilizing communication infrastructure, and a loosely defined coproduced knowledge object [59] (eg, the QF prototype itself). This way, the development of the data collection platform itself is framed in a dynamic, bottom-up, and adaptive way, similar to other open source and peer production experiences.

\section{Conclusions}

Although QF is a project that is still at the prototype stage and with a correspondingly small user base, the cocreation processes of the platform prototype described here represent an example of how the codevelopment of digital research objects, within the relatively new participatory paradigm of extreme citizen science [60], can be implemented following bottom-up, dialogic approaches and a high level of participant engagement. This aligns with the still scarce literature on what has been called do-it-yourself science or peer-to-peer science [61,62], in which similar participatory approaches can offer an opportunity for early and sustained engagement from personal science practitioners in the collaborative definition of concepts, features, and protocols for health-related digital platforms.

\section{Acknowledgments}

The authors would like to thank all participants of the Open Humans community calls and the Quantified Self and OpenCovid19 communities for their input and support. The authors would also like to thank all of the users of Quantified Flu for their support and feedback. The authors are also grateful for the suggestions and recommendations of the two anonymous reviewers who helped to substantially improve our manuscript. Thanks to the Bettencourt Schueller Foundation's long-term partnership, this work was partly supported by the Center for Research and Interdisciplinarity Research Fellowship (awarded to BGT). This work was also supported by a microgrant from the OpenCovid19 initiative. KW was supported by the H2020 WellCo Project (769765).

\section{Authors' Contributions}

BGT, KW, GW, and MB initiated the study. All the authors contributed to the study design. BGT, KA, LB, BM, and MB performed software development. BGT, ESH, IB, and MF performed data analyses and visualizations. BGT, ESH, and MB prepared the original draft of the manuscript. All authors reviewed and edited the manuscript before submission.

\section{Conflicts of Interest}

$\mathrm{MB}$ is the executive director of the Open Humans Foundation. BGT is the director of research for the Open Humans Foundation.

\section{References}

1. Chiauzzi E, Rodarte C, DasMahapatra P. Patient-centered activity monitoring in the self-management of chronic health conditions. BMC Med 2015 Apr 09;13:77 [FREE Full text] [doi: 10.1186/s12916-015-0319-2] [Medline: 25889598]

2. Vayena E, Brownsword R, Edwards SJ, Greshake B, Kahn JP, Ladher N, et al. Research led by participants: a new social contract for a new kind of research. J Med Ethics 2016 Apr;42(4):216-219 [FREE Full text] [doi: 10.1136/medethics-2015-102663] [Medline: 25825527]

3. Allen BL, Ferrier Y, Cohen AK. Through a maze of studies: health questions and 'undone science' in a French industrial region. Environ Sociol 2016 Oct 08;3(2):134-144. [doi: 10.1080/23251042.2016.1220850]

4. Wolf GI, De Groot M. A conceptual framework for personal science. Front Comput Sci 2020 Jun 30;2:00021. [doi: 10.3389/fcomp.2020.00021]

5. White paper: design and implementation of participant-led research in the quantified self community. Quantified Self. URL: https://quantifiedself.com/white-paper-design-and-implementation-of-participant-led-research/ [accessed 2020-09-02]

6. De Groot M, Drangsholt M, Martin-Sanchez F, Wolf G. Single subject (N-of-1) research design, data processing, and personal science. Methods Inf Med 2017;56(6):416-418. [doi: 10.3414/ME17-03-0001] [Medline: 29582912] 
7. Daza EJ, Wac K, Oppezzo M. Effects of sleep deprivation on blood glucose, food cravings, and affect in a non-diabetic: an N-of-1 randomized pilot study. Healthcare (Basel) 2019 Dec 25;8(1):6 [FREE Full text] [doi: 10.3390/healthcare8010006] [Medline: 31881721$]$

8. Lupton D. 'It's made me a lot more aware': a new materialist analysis of health self-tracking. Media Int Aust 2019 Apr 26;171(1):66-79. [doi: 10.1177/1329878x19844042]

9. Heyen NB. From self-tracking to self-expertise: the production of self-related knowledge by doing personal science. Public Underst Sci 2020 Feb;29(2):124-138 [FREE Full text] [doi: 10.1177/0963662519888757] [Medline: 31778095]

10. Mück JE, Ünal B, Butt H, Yetisen AK. Market and patent analyses of wearables in medicine. Trends Biotechnol 2019 Jun;37(6):563-566. [doi: 10.1016/j.tibtech.2019.02.001] [Medline: $\underline{30851983}$ ]

11. Karjalainen J, Viitasalo M. Fever and cardiac rhythm. Arch Intern Med 1986 Jun;146(6):1169-1171. [Medline: 2424378]

12. Kamišalić A, Fister I, Turkanović M, Karakatič S. Sensors and functionalities of non-invasive wrist-wearable devices: a review. Sensors (Basel) 2018 May 25;18(6):1714 [FRE Full text] [doi: 10.3390/s18061714] [Medline: 29799504]

13. Wac K. From quantified self to quality of life. In: Digital Health. Basel, Switzerland: Springer International Publishing; Jan 2018.

14. Clim A, Zota RD, Tinica G. Big Data in home healthcare: a new frontier in personalized medicine. Medical emergency services and prediction of hypertension risks. Int J Healthc Manag 2018 Nov 28;12(3):241-249. [doi: 10.1080/20479700.2018.1548158]

15. Zheng J, Shen Y, Zhang Z, Wu T, Zhang G, Lu H. Emerging wearable medical devices towards personalized healthcare. In: Proceedings of the 8th International Conference on Body Area Networks. 2013 Presented at: 8th International Conference on Body Area Networks; Sep 30-Oct 2, 2013; Boston, United States. [doi: 10.4108/icst.bodynets.2013.253725]

16. Mizuno A, Changolkar S, Patel MS. Wearable devices to monitor and reduce the risk of cardiovascular disease: evidence and opportunities. Annu Rev Med 2021 Jan 27;72:459-471. [doi: 10.1146/annurev-med-050919-031534] [Medline: $\underline{32886543]}$

17. Sana F, Isselbacher EM, Singh JP, Heist EK, Pathik B, Armoundas AA. Wearable devices for ambulatory cardiac monitoring: JACC state-of-the-art review. J Am Coll Cardiol 2020 Apr 07;75(13):1582-1592 [FREE Full text] [doi: 10.1016/j.jacc.2020.01.046] [Medline: $\underline{\text { 32241375] }}$

18. Kourtis LC, Regele OB, Wright JM, Jones GB. Digital biomarkers for Alzheimer's disease: the mobile/ wearable devices opportunity. NPJ Digit Med 2019;2:9 [FREE Full text] [doi: 10.1038/s41746-019-0084-2] [Medline: $\underline{31119198]}$

19. Tyler J, Choi SW, Tewari M. Real-time, personalized medicine through wearable sensors and dynamic predictive modeling: a new paradigm for clinical medicine. Curr Opin Syst Biol 2020 Apr;20:17-25 [FREE Full text] [doi: 10.1016/j.coisb.2020.07.001] [Medline: 32984661$]$

20. Sen-Crowe B, McKenney M, Elkbuli A. Utilizing technology as a method of contact tracing and surveillance to minimize the risk of contracting COVID-19 infection. Am J Emerg Med 2021 Jul;45:519 [FREE Full text] [doi: 10.1016/j.ajem.2020.07.003] [Medline: 32690222]

21. Seshadri DR, Davies EV, Harlow ER, Hsu JJ, Knighton SC, Walker TA, et al. Wearable sensors for COVID-19: a call to action to harness our digital infrastructure for remote patient monitoring and virtual assessments. Front Digit Health 2020 Jun 23;2:8. [doi: 10.3389/fdgth.2020.00008]

22. Jeong H, Rogers JA, Xu S. Continuous on-body sensing for the COVID-19 pandemic: gaps and opportunities. Sci Adv 2020 Sep 2;6(36):eabd4794 [FREE Full text] [doi: 10.1126/sciadv.abd4794] [Medline: 32917604]

23. Bruggeman L. Researchers investigate whether wearable apps could unveil hidden coronavirus cases. ABC News. URL: https://abcnews.go.com/Health/researchers-investigate-wearable-apps-unveil-hidden-coronavirus-cases/story?id=69925541 [accessed 2021-02-06]

24. Quer G, Radin JM, Gadaleta M, Baca-Motes K, Ariniello L, Ramos E, et al. Wearable sensor data and self-reported symptoms for COVID-19 detection. Nat Med 2021 Jan;27(1):73-77. [doi: 10.1038/s41591-020-1123-x] [Medline: 33122860]

25. Mishra T, Wang M, Metwally A, Bogu G, Brooks A, Bahmani A, et al. Early detection of COVID-19 using a smartwatch. medRxiv 2020:20147512 (forthcoming). [doi: 10.1101/2020.07.06.20147512]

26. Natarajan A, Su H, Heneghan C. Assessment of physiological signs associated with COVID-19 measured using wearable devices. NPJ Digit Med 2020 Nov 30;3(1):156 [ [FREE Full text] [doi: 10.1038/s41746-020-00363-7] [Medline: 33299095]

27. Smarr BL, Aschbacher K, Fisher SM, Chowdhary A, Dilchert S, Puldon K, et al. Feasibility of continuous fever monitoring using wearable devices. Sci Rep 2020 Dec 14;10(1):21640 [FREE Full text] [doi: 10.1038/s41598-020-78355-6] [Medline: 33318528]

28. Spinuzzi C. The methodology of participatory design. Tech Commun 2005 May;52(2):163-174 [FREE Full text]

29. Sanders EB, Stappers PJ. Probes, toolkits and prototypes: three approaches to making in codesigning. CoDesign 2014 Mar 06;10(1):5-14. [doi: 10.1080/15710882.2014.888183]

30. Foth M, Axup J. Participatory design and action research: identical twins or synergetic pair? In: Expanding Boundaries in Design: Proceedings Ninth Participatory Design Conference. Canada: Computer Professionals for Social Responsibility; 2006. 
31. Madey G, Freeh V, Tynan R. The open source software development phenomenon: an analysis based on social network theory. In: Proceedings of the Eighth Americas Conference on Information Systems. 2002 Presented at: Eighth Americas Conference on Information System; 2002; Dallas URL: http://melody.syr.edu/hci/amcis02 minitrack/CR/Featherman.pdf

32. Kozinets R. Netnography. In: The International Encyclopedia of Digital Communication and Society. Hoboken, New Jersey: Wiley; Feb 11, 2015.

33. Tzovaras BG, Angrist M, Arvai K, Dulaney M, Estrada-Galiñanes V, Gunderson B, et al. Open Humans: a platform for participant-centered research and personal data exploration. Gigascience 2019 Jun 01;8(6):giz076 [FREE Full text] [doi: 10.1093/gigascience/giz076] [Medline: 31241153]

34. Open Humans Community. Slack. URL: https://slackin.openhumans.org [accessed 2021-02-22]

35. Quantified Self. URL: https://quantifiedself.com [accessed 2021-02-22]

36. Open COVID19 initiative. Just One Giant Lab. URL: https://app.jogl.io/program/opencovid19 [accessed 2021-02-22]

37. OpenHumans / quantified-flu. GitHub. URL: https://github.com/openhumans/quantified-flu [accessed 2021-02-22]

38. Zhao J, Wang T, Fan X. Patient value co-creation in online health communities: Social identity effects on customer knowledge contributions and membership continuance intentions in online health communities. J Serv Manag 2015 Mar 16;26(1):72-96. [doi: 10.1108/josm-12-2013-0344]

39. Costello L, McDermott M, Wallace R. Netnography: range of practices, misperceptions, and missed opportunities. Int $\mathbf{J}$ Qual Methods 2017 Apr 04;16(1):160940691770064. [doi: 10.1177/1609406917700647]

40. Zainal Z. Case study as a research method. J Kemanusiaan 2007;5(1):1 [FREE Full text]

41. Preece J. Etiquette, empathy and trust in communities of practice: stepping-stones to social capital. J Univers Comput Sci 2004;10(3):294-302 [FREE Full text] [doi: 10.3217/jucs-010-03-0294]

42. Quantified Flu. URL: https://quantifiedflu.org [accessed 2021-02-22]

43. OpenHumans / qf-heartrate-apple-health. GitHub. URL: https://github.com/OpenHumans/qf-heartrate-apple-health [accessed 2021-02-22]

44. Mamykina L, Smaldone AM, Bakken SR. Adopting the sensemaking perspective for chronic disease self-management. J Biomed Inform 2015 Aug;56:406-417 [FREE Full text] [doi: 10.1016/j.jbi.2015.06.006] [Medline: 26071681]

45. Hekler EB, Klasnja P, Chevance G, Golaszewski NM, Lewis D, Sim I. Why we need a small data paradigm. BMC Med 2019 Jul 17;17(1):133 [FREE Full text] [doi: 10.1186/s12916-019-1366-x] [Medline: 31311528]

46. Kraut R, Resnick P. Building Successful Online Communities. Cambridge, MA: The MIT Press; 2016.

47. Bot BM, Suver C, Neto EC, Kellen M, Klein A, Bare C, et al. The mPower study, Parkinson disease mobile data collected using ResearchKit. Sci Data 2016 Mar 03;3:160011 [FREE Full text] [doi: 10.1038/sdata.2016.11] [Medline: 26938265]

48. Torous J, Lipschitz J, Ng M, Firth J. Dropout rates in clinical trials of smartphone apps for depressive symptoms: a systematic review and meta-analysis. J Affect Disord 2020 Feb 15;263:413-419. [doi: 10.1016/j.jad.2019.11.167] [Medline: 31969272]

49. Meyerowitz-Katz G, Ravi S, Arnolda L, Feng X, Maberly G, Astell-Burt T. Rates of attrition and dropout in app-based interventions for chronic disease: systematic review and meta-analysis. J Med Internet Res 2020 Sep 29;22(9):e20283 [FREE Full text] [doi: 10.2196/20283] [Medline: $\underline{\text { 32990635] }}$

50. Vaghefi I, Tulu B. The continued use of mobile health apps: insights from a longitudinal study. JMIR Mhealth Uhealth 2019 Aug 29;7(8):e12983 [FREE Full text] [doi: 10.2196/12983] [Medline: 31469081]

51. Call SA, Vollenweider MA, Hornung CA, Simel DL, McKinney WP. Does this patient have influenza? J Am Med Assoc 2005 Feb 23;293(8):987-997. [doi: 10.1001/jama.293.8.987] [Medline: 15728170]

52. Dugas AF, Valsamakis A, Atreya MR, Thind K, Manchego PA, Faisal A, et al. Clinical diagnosis of influenza in the ED. Am J Emerg Med 2015 Jun;33(6):770-775 [FREE Full text] [doi: 10.1016/j.ajem.2015.03.008] [Medline: 25827595]

53. Addeo F, Paoli A, Esposito M, Bolcato M. Doing social research on online communities: The benefits of netnography. Athens J Soc Sci 2019 Dec 05;7(1):9-38. [doi: 10.30958/ajss.7-1-1] [Medline: 22489612]

54. Jong S. Netnography: researching online populations. In: Handbook of Research Methods in Health Social Sciences. Singapore: Springer; 2019.

55. Sack W, Détienne F, Ducheneaut N, Burkhardt J, Mahendran D, Barcellini F. A methodological framework for socio-cognitive analyses of collaborative design of open source software. Comput Supported Coop Work 2006 Jun 10;15(2-3):229-250. [doi: 10.1007/s10606-006-9020-5]

56. Goodyear-Smith F, Jackson C, Greenhalgh T. Co-design and implementation research: challenges and solutions for ethics committees. BMC Med Ethics 2015 Nov 16;16:78 [FREE Full text] [doi: 10.1186/s12910-015-0072-2] [Medline: 26573410]

57. Wiggins A, Wilbanks J. The rise of citizen science in health and biomedical research. Am J Bioeth 2019 Aug 24;19(8):3-14. [doi: 10.1080/15265161.2019.1619859] [Medline: 31339831]

58. Grant AD, Wolf GI, Nebeker C. Approaches to governance of participant-led research: a qualitative case study. BMJ Open 2019 Apr 02;9(4):e025633 [FREE Full text] [doi: 10.1136/bmjopen-2018-025633] [Medline: 30944134]

59. Mahr D, Dickel S. Citizen science beyond invited participation: nineteenth century amateur naturalists, epistemic autonomy, and big data approaches avant la lettre. Hist Philos Life Sci 2019 Oct 07;41(4):41. [doi: 10.1007/s40656-019-0280-z] [Medline: 31591649]

60. Haklay M. Citizen science and volunteered geographic information: overview and typology of participation. In: Crowdsourcing Geographic Knowledge. Dordrecht, Netherlands: Springer; 2013. 
61. Ferretti F. Mapping do-it-yourself science. Life Sci Soc Policy 2019 Jan 14;15(1):1 [FREE Full text] [doi: 10.1186/s40504-018-0090-1] [Medline: 30741364]

62. Delfanti A. Users and peers. From citizen science to P2P science. J Sci Commun 2010;09(01):09010501. [doi: $10.22323 / 2.09010501]$

\section{Abbreviations}

API: application programming interface

OH: Open Humans

QF: Quantified Flu

Edited by R Kukafka; submitted 22.02.21; peer-reviewed by B Smarr, R Cruz Martínez; comments to author 27.04.21; revised version received 16.06.21; accepted 05.07.21; published 10.09.21

Please cite as:

Greshake Tzovaras B, Senabre Hidalgo E, Alexiou K, Baldy L, Morane B, Bussod I, Fribourg M, Wac K, Wolf G, Ball M

Using an Individual-Centered Approach to Gain Insights From Wearable Data in the Quantified Flu Platform: Netnography Study

J Med Internet Res 2021;23(9):e28116

URL: https://www.jmir.org/2021/9/e28116

doi: $10.2196 / 28116$

PMID:

(OBastian Greshake Tzovaras, Enric Senabre Hidalgo, Karolina Alexiou, Lukaz Baldy, Basile Morane, Ilona Bussod, Melvin Fribourg, Katarzyna Wac, Gary Wolf, Mad Ball. Originally published in the Journal of Medical Internet Research (https://www.jmir.org), 10.09.2021. This is an open-access article distributed under the terms of the Creative Commons Attribution License (https://creativecommons.org/licenses/by/4.0/), which permits unrestricted use, distribution, and reproduction in any medium, provided the original work, first published in the Journal of Medical Internet Research, is properly cited. The complete bibliographic information, a link to the original publication on https://www.jmir.org/, as well as this copyright and license information must be included. 